\title{
A Direct Solution for Blind Separation of Sources
}

\author{
A. Mansour, C. Jutten * \\ INPG-TIRF \\ 46, avenue Félix Viallet \\ 38031 Grenoble Cedex \\ Tel. +33765748 fax +3376574790 \\ Email: chris@tirf.inpg.fr \\ EDICS SP 3.2..
}

IEEE Trans. on Signal Processing Vol 44 N3 March 1996

\section{Contents}

1 Introduction $\quad 2$

2 Model equations $\quad 3$

2.1 Mixture model . . . . . . . . . . . . . . . . . 3

2.2 Moments and cumulants . . . . . . . . . . . . . . . 3

3 Mixture matrix estimation 3

3.1 System of equations based on cross-moments . . . . . . . . . . 4

3.2 System of equation based on cross-cumulants . . . . . . . . . . . 4

4 Gaussian sources $\quad 5$

4.1 The source signals are Gaussian . . . . . . . . . . . . 5

4.2 One of the sources is Gaussian . . . . . . . . . . 5

5 The sources are not Gaussian $\quad 6$ 
$5.1 \quad$ Particular case $m_{11} m_{22}=-m_{12} m_{21} \ldots \ldots \ldots \ldots \ldots$

5.2 General case . . . . . . . . . . . . . . . . . . 7

5.2.1 Separation with the 4 th-order cumulants . . . . . . . 8

$5.2 .2 \quad$ A simpler solution . . . . . . . . . . . . . . 9

6 Robustness of the method $\quad 9$

6.1 Error influence . . . . . . . . . . . . . . . . . . 9

6.2 Improvement condition . . . . . . . . . . . . . . 10

6.3 Experimental results . . . . . . . . . . . . . . . . 11

$\begin{array}{lll}7 & \text { Conclusion } & 12\end{array}$

\begin{abstract}
The paper proposes a direct estimation of the mixing matrix and not a tuning of the separation matrix as usually done. The method is based on resolution of a set of 8 equations involving cumulants up to order 4 of the mixture signals, which may be reduce to resolution to a second order polynomial equation.

Experimental results and robustness suggest to use the method as a preliminary step to propose good starting points.
\end{abstract}

\title{
1 Introduction
}

Most of the recent solutions for blind separation of sources are based on estimation of a separation matrix, such that the product of the observation signals (mixtures) vector multiplied by the matrix gives statistically independent signals. The algorithms, adaptive or not, use an independence criterion based on higher-order statistics (moments or rather cumulants). Methods include minimization of independence criteria ([4], [3], [9]) or contrast functions ([5], [10]) or cancellation of multiple criteria ([8], [12]).

In this paper, we propose a direct method, which consists in directly estimating the mixture matrix (and not a separation matrix) from the observation of the mixtures. The problem is restricted to instantaneous mixtures of two sources as described in section 2. The sets of equations, based on 4th-order cross-moments and cross-cumulants, are derived in the section 3, and discussed 
in the case of Gaussian signals and in the general case in sections 4 and 5, respectively. The precision of the method and experimental results are given in the section 7 , before the conclusion.

\section{Model equations}

\section{$2.1 \quad$ Mixture model}

At any time $t$, we observe, with help of two sensors, two instantaneous mixtures $e_{i}(t)$ of the two zero-mean sources $x_{i}(t)$, assumed statistically independent. Denoting $M$ the unknown mixture matrix with real coefficients, we have:

$$
\left(\begin{array}{l}
e_{1}(t) \\
e_{2}(t)
\end{array}\right)=\left(\begin{array}{ll}
m_{11} & m_{12} \\
m_{21} & m_{22}
\end{array}\right)\left(\begin{array}{l}
x_{1}(t) \\
x_{2}(t)
\end{array}\right) .
$$

\subsection{Moments and cumulants}

Let us denote the cross-moments and the cross-cumulants of the observations:

$$
\begin{aligned}
\operatorname{Mom}_{k l}\left(e_{1}, e_{2}\right) & =E\left[e_{1}^{k}(t) e_{2}^{l}(t)\right], \\
\operatorname{Cum}_{k l}\left(e_{1}, e_{2}\right) & =\operatorname{Cum}\left(e_{1}^{k}(t) e_{2}^{l}(t)\right)=C_{k l},
\end{aligned}
$$

and the unknown moments and cumulants of the sources:

$$
\begin{aligned}
p_{i} & =E\left[x_{i}^{2}(t)\right], \\
\gamma_{i} & =E\left[x_{i}^{4}(t)\right], \\
\beta_{i} & =\operatorname{Cum}\left(x_{i}^{4}\right)=\gamma_{i}-3 p_{i} .
\end{aligned}
$$

\section{Mixture matrix estimation}

We know that the necessary information for separate our sources are in the mixture statistics. The various cross-moments and cross-cumulants of the mixture signals can be expanded, using (1), with respect to the coefficients of the mixture matrix and source statistics. Equating each equation to the estimated cross-moments or cross-cumulants leads to a system of non-linear equations. Can we compute the mixture matrix directly by solving the nonlinear system ?

In indirect methods of source separation, it has been proved that while 2ndorder statistics are generally not sufficient exception special cases ([14], [6], [11]), 4th-order statistics are adequate. However, is it better to derive equations based on cross-moments or on cross-cumulant? 


\subsection{System of equations based on cross-moments}

From the relation (1), we can express cross-moments of the mixtures $e_{1}(t)$ and $e_{2}(t)$ with respect to the coefficients $m_{i j}$, cross-moments and cross-cumulants of the sources $x_{1}(t)$ and $x_{2}(t)$. Then, up to 4th-order, taking into account the statistical independence of the sources, we get the following system of 8 nonlinear equations of 8 variables $m_{i j}, p_{i}, \gamma_{i},(i, j \in[1,2])$ :

$$
\begin{aligned}
\operatorname{Mom}_{11}\left(e_{1}, e_{2}\right)= & m_{11} m_{21} p_{1}+m_{12} m_{22} p_{2}, \\
\operatorname{Mom}_{20}\left(e_{1}, e_{2}\right)= & m_{11}^{2} p_{1}+m_{12}^{2} p_{2}, \\
\operatorname{Mom}_{02}\left(e_{1}, e_{2}\right)= & m_{21}^{2} p_{1}+m_{22}^{2} p_{2}, \\
\operatorname{Mom}_{31}\left(e_{1}, e_{2}\right)= & 3 m_{11} m_{12}\left(m_{11} m_{22}+m_{21} m_{12}\right) p_{1} p_{2} \\
& +m_{11}^{3} m_{21} \gamma_{1}+m_{12}^{3} m_{22} \gamma_{2}, \\
\operatorname{Mom}_{13}\left(e_{1}, e_{2}\right)= & 3 m_{21} m_{22}\left(m_{11} m_{22}+m_{21} m_{12}\right) p_{1} p_{2} \\
& +m_{11} m_{21}^{3} \gamma_{1}+m_{12} m_{22}^{3} \gamma_{2}, \\
\operatorname{Mom}_{22}\left(e_{1}, e_{2}\right)= & \left(m_{11}^{2} m_{22}^{2}+4 m_{11} m_{21} m_{12} m_{22}+m_{12}^{2} m_{21}^{2}\right) p_{1} p_{2} \\
& +m_{11}^{2} m_{21}^{2} \gamma_{1}+m_{12}^{2} m_{22}^{2} \gamma_{2}, \\
\operatorname{Mom}_{40}\left(e_{1}, e_{2}\right)= & m_{11}^{4} \gamma_{1}+6 m_{11}^{2} m_{12}^{2} p_{1} p_{2}+m_{12}^{4} \gamma_{2}, \\
\operatorname{Mom}_{04}\left(e_{1}, e_{2}\right)= & m_{21}^{4} \gamma_{1}+6 m_{21}^{2} m_{22}^{2} p_{1} p_{2}+m_{22}^{4} \gamma_{2} .
\end{aligned}
$$

In fact, even with the help of tools like Mathematica, we do not succeed in solving the system. Then we propose to study the system based on the crosscumulants.

\subsection{System of equation based on cross-cumulants}

According to a similar approach, the cross-cumulants of the mixture signals, up to the 4 th-order, leads to the following system of 8 nonlinear equations:

$$
\begin{aligned}
& C_{11}=\operatorname{Cum}_{11}\left(e_{1}, e_{2}\right)=m_{11} m_{21} p_{1}+m_{12} m_{22} p_{2}, \\
& C_{20}=\operatorname{Cum}_{20}\left(e_{1}, e_{2}\right)=m_{11}^{2} p_{1}+m_{12}^{2} p_{2}, \\
& C_{02}=\operatorname{Cum}_{02}\left(e_{1}, e_{2}\right)=m_{21}^{2} p_{1}+m_{22}^{2} p_{2}, \\
& C_{31}=\operatorname{Cum}_{31}\left(e_{1}, e_{2}\right)=m_{11}^{3} m_{21} \beta_{1}+m_{12}^{3} m_{22} \beta_{2}, \\
& C_{13}=\operatorname{Cum}_{13}\left(e_{1}, e_{2}\right)=m_{11} m_{21}^{3} \beta_{1}+m_{12} m_{22}^{3} \beta_{2}, \\
& C_{22}=\operatorname{Cum}_{22}\left(e_{1}, e_{2}\right)=m_{11}^{2} m_{21}^{2} \beta_{1}+m_{12}^{2} m_{22}^{2} \beta_{2}, \\
& C_{40}=\operatorname{Cum}_{40}\left(e_{1}, e_{2}\right)=m_{11}^{4} \beta_{1}+m_{12}^{4} \beta_{2}, \\
& C_{04}=\operatorname{Cum}_{04}\left(e_{1}, e_{2}\right)=m_{21}^{4} \beta_{1}+m_{22}^{4} \beta_{2},
\end{aligned}
$$

We note the formal expressions of the equations are quite simple. In the following, we will derive the analytical solutions of the system in many situations. 


\section{Gaussian sources}

\subsection{The source signals are Gaussian}

If the 2 source signals are Gaussian, then their kurtosis are equal to zero. In this case, we cannot separate the source signal. In fact, the relations (18) to (22) are equal to zero. Then we just have three equations of six variables (the matrix coefficients $m_{i j}$ and the source powers $p_{i}$ ), and we need more information to solve the system. This result is in accordance with well known results on separation of Gaussian source [5].

\subsection{One of the sources is Gaussian}

If only one of the two sources is Gaussian (for example $x_{1}(t)$ ), then its 4th-order cumulant will be equal to zero $\left(\beta_{1}=0\right)$. Equations $(18)$ to $(22)$ then become:

$$
\begin{aligned}
& C_{11}=\operatorname{Cum}_{11}\left(e_{1}, e_{2}\right)=m_{21} p_{1}+m_{12} p_{2}, \\
& C_{20}=\operatorname{Cum}_{20}\left(e_{1}, e_{2}\right)=m_{11}^{2} p_{1}+m_{12}^{2} p_{2}, \\
& C_{02}=\operatorname{Cum}_{02}\left(e_{1}, e_{2}\right)=m_{21}^{2} p_{1}+m_{22}^{2} p_{2}, \\
& C_{31}=\operatorname{Cum}_{31}\left(e_{1}, e_{2}\right)=m_{12}^{3} m_{22} \beta_{2}, \\
& C_{13}=\operatorname{Cum}_{13}\left(e_{1}, e_{2}\right)=m_{12} m_{22}^{3} \beta_{2}, \\
& C_{22}=\operatorname{Cum}_{22}\left(e_{1}, e_{2}\right)=m_{12}^{2} m_{22}^{2} \beta_{2}, \\
& C_{40}=\operatorname{Cum}_{40}\left(e_{1}, e_{2}\right)=m_{12}^{4} \beta_{2}, \\
& C_{04}=\operatorname{Cum}_{04}\left(e_{1}, e_{2}\right)=m_{22}^{4} \beta_{2},
\end{aligned}
$$

The five equations (26) to (30) only involving 3 variables, it is simple to prove we cannot find the 4 coefficients $m_{i j}$. We know the separation of the sources is possible up to a coefficient, then we always may suppose that the diagonal coefficients of the mixture matrix are equal to one: $m_{i i}=1$. Moreover, we assume the mixture matrix is not singular: $1-m_{12} m_{21} \neq 0$.

Then, using the assumptions $m_{i i}=1$, from relations (30) and (27), we find the value of $\beta_{2}$ and $m_{12}$ :

$$
\begin{aligned}
\beta_{2} & =C_{04} \neq 0 \\
m_{12} & =\frac{C_{13}}{C_{04}} .
\end{aligned}
$$

The three relations (26), (28) and (29) carry any supplementary information, but we can use the relations to detect the situation. From the first three relations (23) to (25), we find the other variables: 


$$
\begin{aligned}
m_{21} & =\frac{C_{11}-C_{02} m_{12}}{C_{20}-C_{11} m_{12}}, \\
p_{2} & =\frac{C_{02}-m_{21}^{2} C_{20}}{1-m_{12}^{2} m_{21}^{2}}, \\
p_{1} & =C_{20}-m_{12}^{2} p_{2} .
\end{aligned}
$$

From the solutions, it is easy to deduce the sources by multiplying the mixture vector signal by the inverse of estimated mixture matrix.

\section{The sources are not Gaussian}

We suppose in this section that the mixture matrix is not a singular matrix $\left(m_{11} m_{22}-m_{12} m_{21} \neq 0\right)$. Considering the system (18) to (22), we can find the general solution under the condition $m_{11} m_{22} \neq \pm m_{12} m_{21}$. The condition first leads to $m_{11} m_{22} \neq m_{12} m_{21}$ : this means the mixture matrix is not singular. In that case, clearly the separation cannot be achieved.

The condition also leads to the special case $m_{11} m_{22} \neq-m_{12} m_{21}$. We will study that case in the next subsection.

In the following, we still suppose $m_{i i}=1$, because the solution is up to any diagonal matrix and up to any permutation matrix.

\subsection{Particular case $m_{11} m_{22}=-m_{12} m_{21}$}

With $m_{11}=m_{22}=1$, the condition becomes $1=-m_{12} m_{21}$, that is the mixture matrix has the following form:

$$
M=\left(\begin{array}{cc}
1 & -\frac{1}{m} \\
m & 1
\end{array}\right) .
$$

Solving the nonlinear system leads to:

$$
\begin{aligned}
& m_{21}= \pm \sqrt{\frac{C_{02}}{C_{20}}}, \\
& m_{22}=-\frac{1}{m_{21}} .
\end{aligned}
$$

The above solutions emphasizes on a sign indetermination, which gives 2 pairs of solutions. The other parameters (sources statistics) can also be analytically 
computed. From a practical point of view, the special case can be detected using:

$$
\frac{C_{20}}{C_{02}}=\frac{C_{31}}{C_{13}}=\frac{C_{40}}{C_{22}}=\frac{C_{22}}{C_{04}}
$$

\subsection{General case}

Now we suppose that we have $m_{11} m_{22} \neq \pm m_{12} m_{21}$. Then, it is quite easy to prove the system of 8 equations, (15) to (22), only consists of 6 independent equations of 8 variables. Again, it proves the 4 mixture coefficients cannot be found. In the following, we will then assume $m_{11}=m_{22}=1$.

From the 5 relations $(15),(16),(17),(21)$ and (22), we especially can derive the mixture coefficient $m_{12}$ and the source statistics with respect to the other mixture coefficient, and estimations of mixture cross-cumulants $C_{i j}$ :

$$
\begin{aligned}
m_{12} & =\frac{C_{11}-C_{20} m_{21}}{C_{02}-C_{11} m_{21}}, \\
p_{1} & =\frac{C_{20}-C_{02} m_{12}^{2}}{1-m_{12}^{2} m_{21}^{2}}, \\
p_{2} & =\frac{C_{02}-C_{20} m_{21}^{2}}{1-m_{12}^{2} m_{21}^{2}}, \\
\beta_{1} & =\frac{C_{40}-C_{04} m_{12}^{4}}{1-m_{12}^{4} m_{21}^{4}}, \\
\beta_{2} & =\frac{C_{04}-C_{40} m_{21}^{4}}{1-m_{12}^{4} m_{21}^{4}} .
\end{aligned}
$$

Clearly, the relation (40) defines an infinite number of points $\left(m_{12}, m_{21}\right)$ belonging to an hyperbola. Using the relations (40) ...(44) in (18) ...(20), we get:

$$
\begin{aligned}
\left(C_{02}^{2} C_{22}\right. & \left.-C_{11}^{2} C_{04}\right)+2 m_{21} C_{11}\left(C_{20} C_{04}-C_{02} C_{22}\right) \\
& +m_{21}^{2}\left(2 C_{11}^{2} C_{22}-C_{20}^{2} C_{04}-C_{02}^{2} C_{40}\right) \\
& +2 m_{21}^{3} C_{11}\left(C_{02} C_{20}-C_{20} C_{22}\right)+m_{21}^{4}\left(C_{22} C_{20}^{2}-C_{11}^{2} C_{40}\right)=0(45)
\end{aligned}
$$

The relation (45) is a fourth degree polynomial equation of the variable $m_{21}$. The equation has a nice property: suppose that the $m_{21}^{\star}$ is a root of that equation, then $\frac{1}{m_{21}^{\star}}$ is also a root. This property has already been proved by Sorouchyari [13] and Fort [7]. Finally, solving the equation (45) is possible with various classical methods and gives $m_{21}$. Then replacing in (40) gives $m_{12}$.

\footnotetext{
${ }^{1}$ It is easy to prove that $m_{11} m_{22} \neq \pm m_{12} m_{21} \Longrightarrow C_{02}-C_{11} m_{21} \neq 0$.
} 


\subsubsection{Separation with the 4 th-order cumulants}

Up to now, to estimate the mixture coefficients, we use cross-cumulants up to 4th-order. In this section, we wonder if the use of only fourth order cumulants is enough.

Assuming $m_{11}=m_{22}=1$, we then have 5 equations of 4 variables, $\left(m_{12}\right.$, $m_{21}, \beta_{1}$ and $\left.\beta_{2}\right)$ :

$$
\begin{aligned}
& C_{31}=m_{21} \beta_{1}+m_{12}^{3} \beta_{2}, \\
& C_{13}=m_{21}^{3} \beta_{1}+m_{12} \beta_{2}, \\
& C_{22}=m_{21}^{2} \beta_{1}+m_{12}^{2} \beta_{2}, \\
& C_{40}=\beta_{1}+m_{12}^{4} \beta_{2}, \\
& C_{04}=m_{21}^{4} \beta_{1}+\beta_{2} .
\end{aligned}
$$

We first assume the condition $m_{12} m_{21} \neq \pm 1$ holds, otherwise we may refer to results of section 5.1. We also assume sources are not Gaussian, that is $\beta_{1} \beta_{2} \neq 0$.

Now, assuming $m_{12} m_{21} \neq 0$, from the two relations (49) and (50):

$$
\begin{aligned}
& \beta_{1}=\frac{C_{40}-C_{04} m_{12}^{4}}{1-m_{21}^{4} m_{12}^{4}}, \\
& \beta_{2}=\frac{C_{04}-C_{40} m_{21}^{4}}{1-m_{21}^{4} m_{12}^{4}} .
\end{aligned}
$$

Replacing in (46) to (50) leads to:

$$
\begin{aligned}
C_{31}\left(1+m_{21}^{2} m_{12}^{2}\right)\left(1+m_{21} m_{12}\right) & =C_{40} m_{21}\left(1+m_{21} m_{12}+m_{21}^{2} m_{12}^{2}\right)+C_{04} m_{12}^{3} \\
C_{13}\left(1+m_{21}^{2} m_{12}^{2}\right)\left(1+m_{21} m_{12}\right) & =C_{40} m_{21}^{3}+C_{04} m_{12}\left(1+m_{21} m_{12}+m_{21}^{2} m_{12}^{2}\right) \\
C_{22}\left(1+m_{21}^{2} m_{12}^{2}\right) & =C_{40} m_{21}^{2}+C_{04} m_{12}^{2}
\end{aligned}
$$

From (55) and (54), we derive:

$$
m_{12}=\frac{C_{13}-C_{22} m_{21}}{C_{04}-C_{13} m_{21}} .
$$

It is easy to check that $C_{04}-C_{13} m_{21}=\beta_{2}\left(1-m_{12} m_{21}\right)$, so if $C_{04}-C_{13} m_{21} \neq$ 0 except if one source (at least) is Gaussian or if the mixture matrix is singular.

Finally, using (56) in the equation (55):

$$
\begin{aligned}
\left(C_{40} C_{13}^{2}-C_{22}^{3}\right) m_{21}^{4}+2 C_{13}\left(C_{22}^{2}-C_{40} C_{04}\right) m_{21}^{3} & +\left(C_{40} C_{04}^{2}+C_{04} C_{22}^{2}-2 C_{22} C_{13}^{2}\right) m_{21}^{2} \\
& +C_{04}\left(C_{13}^{2}-C_{22} C_{04}\right)=0 .
\end{aligned}
$$

As in the section 5.2, we find again a fourth order polynomial equation. 


\subsubsection{A simpler solution}

We still suppose $m_{i i}=1$ and $m_{12} m_{21} \neq \pm 1$. Using the three2nd order crosscumulants:

$$
\begin{aligned}
& C_{11}=p_{1} m_{21}+p_{2} m_{12}, \\
& C_{20}=p_{1}+m_{12}^{2} p_{2}, \\
& C_{02}=p_{1} m_{21}^{2}+p_{2},
\end{aligned}
$$

leads to another relation between $m_{12}$ and $m_{21}$, which again is an hyperbola, like (56):

$$
m_{12}=\frac{C_{11}-C_{20} m_{21}}{C_{02}-C_{11} m_{21}} .
$$

satisfying simultaneously the relations (56) and the relation (58), we find the value of $m_{21}$ with a simple second degree equation:

$$
\left(C_{13} C_{20}-C_{22} C_{11}\right) m_{21}^{2}+\left(C_{02} C_{22}-C_{04} C_{20}\right) m_{21}+C_{11} C_{04}-C_{13} C_{02}=0 .
$$

The two roots of the equation correspond to the solution of the separation problem up to a permutation.

\section{Robustness of the method}

\subsection{Error influence}

In this section, we still suppose that $m_{11}=m_{22}=1$. Let us denote the estimation errors by: $d_{1}=d\left[\operatorname{Cum}_{11}\left(e_{1}, e_{2}\right)\right], d_{2}=d\left[\operatorname{Cum}_{20}\left(e_{1}, e_{2}\right)\right], d_{3}=$ $d\left[\operatorname{Cum}_{02}\left(e_{1}, e_{2}\right)\right], d_{4}=d\left[\operatorname{Cum}_{31}\left(e_{1}, e_{2}\right)\right], d_{5}=d\left[\operatorname{Cum}_{13}\left(e_{1}, e_{2}\right)\right]$, and $d_{6}=$ $d\left[\operatorname{Cum}_{22}\left(e_{1}, e_{2}\right)\right]$. The influence of estimation errors on the results is given by a linear system that we may directly derive from the nonlinear system of the section 3.2 by a simple differentiation:

$$
\left[\begin{array}{cccccc}
m_{21} & p_{1} & p_{2} & m_{12} & 0 & 0 \\
1 & 0 & 2 m_{12} p_{2} & m_{12}^{2} & 0 & 0 \\
m_{21}^{2} & 2 m_{21} p_{1} & 0 & 1 & 0 & 0 \\
0 & \beta_{1} & 3 \beta_{2} m_{12}^{2} & 0 & m_{21} & m_{12}^{3} \\
0 & 3 \beta_{1} m_{21}^{2} & \beta_{2} & 0 & m_{21}^{3} & m_{12} \\
0 & 2 \beta_{1} m_{21} & 2 m_{12} \beta_{2} & 0 & m_{21}^{2} & m_{12}^{2}
\end{array}\right]\left[\begin{array}{c}
d p_{1} \\
d m_{21} \\
d m_{12} \\
d p_{2} \\
d \beta_{1} \\
d \beta_{2}
\end{array}\right]=\left[\begin{array}{c}
d_{1} \\
d_{2} \\
d_{3} \\
d_{4} \\
d_{5} \\
d_{6}
\end{array}\right] .
$$


For sake of simplicity, we will suppose that the cumulants of the same order have the same estimation error. $d_{1}=d_{2}=d_{3}=\delta_{2}$ and $d_{4}=d_{5}=d_{6}=\delta_{4}$. The solution of the system (60) especially provides:

$$
\begin{aligned}
\frac{d m_{21}}{m_{21}} & =\frac{\left(1-m_{12}\right)\left(1-m_{21}\right)\left(p_{2} \delta_{4}-\beta_{2} m_{12} \delta_{2}\right)}{m_{21}\left(m_{12} m_{21}-1\right)\left(\beta_{2} m_{12} p_{1}-\beta_{1} m_{21} p_{2}\right)}, \\
\frac{d m_{12}}{m_{12}} & =\frac{\left(p_{1} \delta_{4}-\beta_{1} m_{21} \delta_{2}\right)\left(m_{21}+m_{12}-1-m_{12} m_{21}\right)}{m_{12}\left(m_{12} m_{21}-1\right)\left(\beta_{2} m_{12} p_{1}-\beta_{1} m_{21} p_{2}\right)} .
\end{aligned}
$$

Unfortunately, the above expressions are not practically relevant.

\subsection{Improvement condition}

Another approach to the robustness study is to find the condition witch is uses to improve the residual crosstalk. Let us suppose that the mixture matrix is

$$
M=\left[\begin{array}{ll}
1 & a \\
b & 1
\end{array}\right]
$$

and estimated mixture matrix:

$$
\hat{M}=\left[\begin{array}{ll}
1 & x \\
y & 1
\end{array}\right] .
$$

Finally, the sources are estimated by the global matrix $G$ :

$$
G=\hat{M}^{-1} M=\left[\begin{array}{cc}
\frac{1-x b}{1-x y} & \frac{a-x}{1-x y} \\
\frac{b-y}{1-x y} & \frac{1-a y}{1-x y}
\end{array}\right] .
$$

The solution of the blind separation of sources is estimated up to coefficient, then we may compute the crosstalk improvement on normalized output signals. The normalized global matrix will give us four possible similar cases. Then, we will study that one:

$$
G_{N}=\left[\begin{array}{cc}
1 & \frac{a-x}{1-x y} \\
\frac{b-y}{1-a y} & 1
\end{array}\right] \text {. }
$$

So, to improve the residual crosstalk, the following condition must be verified:

$$
\left|\frac{a-x}{1-x b}\right|<|a| \Longleftrightarrow x(1-a b)(x+x a b-2 a)<0 .
$$

We have a similar condition on $y$. Let us denote the estimation error by $d$. Then we can deduce from (66) another condition according to $d$ :

$$
d^{2}\left(1-a^{2} b^{2}\right)+2 a^{2} b d(1-a b)-a^{2}(1-a b)^{2}<0
$$


For sake of simplicity, we will discuss the only case ${ }^{2}$ where $|a b|<1$. The solution of $(67)$ is :

$$
\min \left[-a, a \frac{1-a b}{1+a b}\right]<d<\max \left[-a, a \frac{1-a b}{1+a b}\right] .
$$

For study the condition (68) according to the relative estimation error $d / a$, we will suppose that $a>0^{3}$, then this condition will be:

$$
-1<\frac{d}{a}<\frac{1-a b}{1+a b}
$$

Finally, let us denote the relative estimation error of the second order cumulant by $\delta_{2}^{r}$ and the fourth order one by $\delta_{4}^{r}$. From the relation (61) and the condition (69), we will find that :

$$
\begin{aligned}
\delta_{2}^{r} m_{12}-\frac{m_{21}\left(1-m_{12} m_{21}\right)^{2}}{\left(1-m_{12}\right)\left(1-m_{21}\right)\left(1+m_{12} m_{21}\right)} & \leq \delta_{4}^{r} \\
\delta_{2}^{r} m_{12}+\frac{m_{21}\left(m_{12}-m_{21}\right)\left(1-m_{21} m_{21}\right)}{\left(1-m_{12}\right)\left(1-m_{21}\right)} & \geq \delta_{4}^{r} .
\end{aligned}
$$

It means the improvement condition is satisfied if the estimation of 4 th order cross-cumulants accurate enough with respect to 2 nd order ones. For a given number of samples, $\delta_{2}^{r}$ and $\delta_{4}^{r}$ are fixed ( see [2]), and then the condition depends of the mixing matrix,. The improvement is then bounded, with respect the mixing matrix.

Each estimation of $\hat{M}$ allow to improve the separation. We can then iterate this step on the output signals $s=G x$.

\subsection{Experimental results}

In this section, we used the simple solution of paragraph 5.2.2. The estimation of the different cumulants ${ }^{4}$ of the mixture signals, is done with help of the algorithm proposed by Amblard et al. [1]. The algorithm is efficient but only when our signals are stationary (see [1] and [2]).

If the sources are i.i.d signals, then we get about $-22 \mathrm{~dB}$ for the residual crosstalk (see Fig. 1) using statistics estimated over about 500 samples.

We also tested the method with non stationary signals: one of the signals is a speech signal (a part of the word "Bonjour") and the other one is an i.i.d signal. We found about $-20 \mathrm{~dB}$ for the residual crosstalk, if the sample number

\footnotetext{
2 the others cases are similars

${ }^{3}$ Now, we have the condition $a|b|<1$

${ }^{4}$ The estimation depends on sample number
} 
is 500 .

The shape of the signals : observations, estimated sources and estimation errors are shown on Fig. 2.

In the general case of non stationary signals, the estimation must be done on a few number of samples. For instance, we estimate the cumulant over only 100 samples. After five iterations as proposed in paragraph 6.2, we got about $-17 \mathrm{~dB}$ for the residual crosstalk with i.i.d. sources (see Fig. 3.a). With similar estimation in the case of non stationary signals (speech signals), we obtained a bad result (see Fig. 2.b).

\section{Conclusion}

In this paper, we first proved that equations based on cumulants are simpler to derive than these based on cross-moments. We proposed a direct method, based on cumulants estimation and on the resolution of a 2 nd degree polynomial equation. We also proved the method may be applied in any case, except if the two sources are Gaussian. The method is then quite simple, but the solutions obtained are very sensitive to the precision of the estimated cumulants.

If the sources are stationary, good results can be obtained by estimating statistics over 500 samples or more. However, in the case of non stationary signals, the number of samples must be decreased, and relevant improvement of the crosstalk is more difficult. A solution can be to repeat the process as suggested in the paragraph 6.2.

Here, we restricted the study to the separation of 2 sources, but theoretical solutions for more than 3 sources are not easily tractable.

The direct solution seems finally not so precise than indirect methods, especially adaptive algorithms. However, it can give, for such an algorithm, a good starting point with a small computation cost, and then allow a faster convergence.

\section{References}

[1] P. O. Amblard and J. M. Brossier. Adaptative estimation of the fourthorder cumulant of an i.i.d. stochastic process. Signal Processing, 42:37-43, 


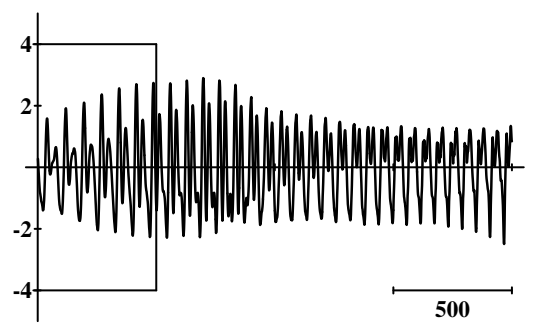

(a) Source $x_{1}(t)$

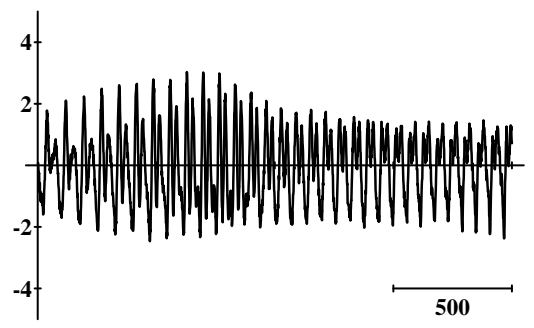

(c) output $s_{1}(t)$;

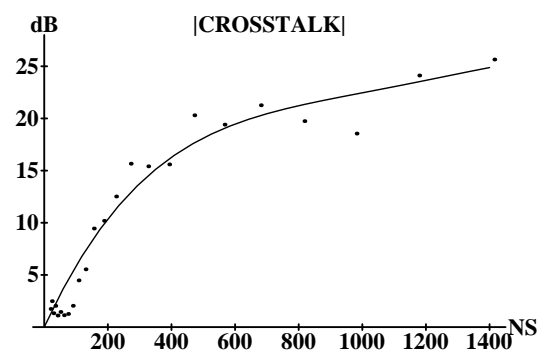

(e) absolute value of the crosstalk

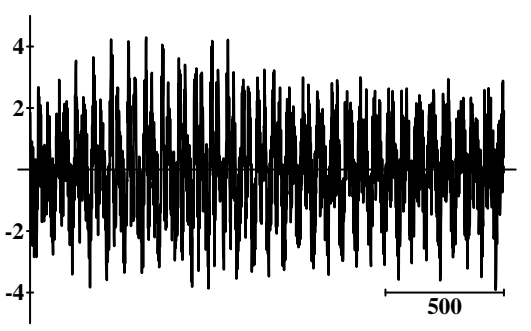

(b) mixture $e_{1}(t)$;

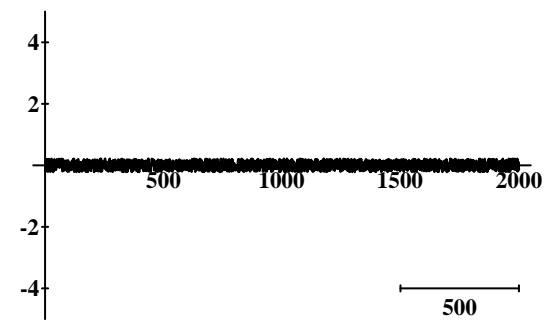

(d) error signal $s_{1}(t)-x_{1}(t)$;

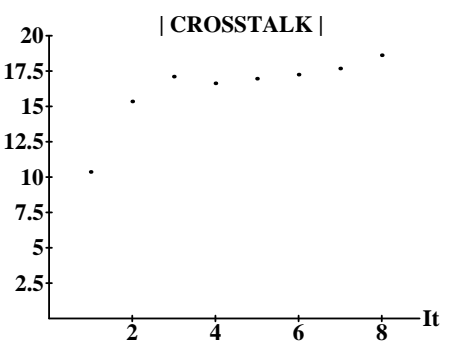

absolute value of the crosstalk after iterative realisations

Figure 1: Signals and performance results. 
February 1995 .

[2] A. Benvéniste, M. Métivier, and P. Priouret. Adaptive algorithms and stochastic approximations. Spring-Verlag, 1990.

[3] J. F. Cardoso. Blind identification of independent signals. In Workshop on Higher-Order Spectral Analysis, Vail (CO), USA, June 1989.

[4] P. Comon. Separation of sources using higher-order cumulants. In SPIE Vol. 1152 Advanced Algorithms and Architectures for Signal Processing IV, San Diego (CA), USA, August 8-10 1989.

[5] P. Comon. Independent component analysis, a new concept? Signal Processing, 36(3):287-314, April 1994.

[6] L. Féty. Méthodes de traitement d'antenne adaptées aux radiocommunications. PhD thesis, ENST Paris, 1988.

[7] J. C. Fort. Stabilité de l'algorithme de séparation de sources de Jutten et Hérault. Traitement du signal, 8(1):35-42, 1991.

[8] C. Jutten and J. Hérault. Blind separation of sources, Part I: An adaptive algorithm based on a neuromimetic architecture. Signal Processing, 24(1):1-10, 1991.

[9] J. L. Lacoume and P. Ruiz. Sources identification: A solution based on cumulants. In IEEE ASSP WorkshopV, Mineapolis, USA, August 1988.

[10] E. Moreau and O. Macchi. New self-adaptive algorithms for source separation based on contrast functions. In IEEE Signal Processing Workshop on Higher-Order Statistics, pages 215-219, South Lac Tahoe, USA (CA), June 1993.

[11] D. T. Pham and P. Garat. Séparation aveugle de sources temporellement corrélées. In Actes du XIVème colloque GRETSI, pages 317-320, JuanLes-Pins, France, September 1993.

[12] D. T. Pham, P. Garat, and C. Jutten. Separation of a mixture of independent sources through a maximum likelihood approach. In J. Vandewalle, R. Boite, M. Moonen, and A. Oosterlinck, editors, Signal Processing VI, Theories and Applications (EUSIPCO'92), pages 771-774, Brussels, Belgium, August 1992. Elsevier.

[13] E. Sorouchyari. Blind separation of sources, Part III: Stability analysis. Signal Processing, 24(1):21-29, 1991.

[14] R. L. L. Tong and Y. H. V. C. Soon. Indeterminacy and identifiability of blind identification. IEEE Trans. on CAS, 38:499-509, May 1991. 